\title{
Energy-Mass Duality of Heat and Its Applications
}

\author{
Zengyuan Guo*
}

Heat conduction in a medium can be modelled as the motion of a weighty phonon gas in a dielectric based on the concept of thermomass. Newtonian mechanics has then been used to establish the momentum equation for the phonon gas, which is the general conduction law and it degenerates into various heat conduction models for the appropriate simplified conditions. These phenomena show that heat has energymass duality, that is, heat acts like energy during its conversion with other forms of energy and then acts like mass during its motion. Furthermore, the general relation between the heat flux and the temperature gradient can be derived from the Boltzmann transport equation for phonons. In the high heat flux case the thermal conductivity for nano-materials calculated based on Fourier's law is the apparent thermal conductivity, which is less than the actual intrinsic thermal conductivity. A more general heat conduction model for nano-systems is then presented. Finally, the quantity, entransy, is introduced based on an analogy between heat conduction and electric conduction, which is a simplified expression of the thermomass potential energy. The principle of minimum entransy dissipation-based thermal resistance can be used for optimizing the heat transfer process to increase the energy efficiency.

Keywords: Thermomass theory; General heat conduction law; Non-Fourier heat conduction; Entransy

Received 2 September 2018, Accepted 23 September 2018

DOI: $10.30919 /$ esee $8 \mathrm{c} 146$

\section{Introduction}

Heat transfer, a branch of general physics, has been well developed and widely applied in various engineering fields. However, the development of new high-tech applications has presented many new challenges for the heat transfer discipline. For example, since the frequencies of lasers used in weapons and for material processing have reached picosecond to femtosecond order of magnitudes, the Fourier heat conduction law, the core heat transfer law, is no longer applicable with ultra-fast heating of various objects. Another example is that the Fourier heat conduction law breaks down for heat conduction problems in nano-materials and carbon nanotubes because the size effect cannot be neglected in the nano-scale.

In fact, in the early 1950s, the Fourier heat conduction law was questioned, because Fourier's law leads to the nonphysical conclusion for transient heat conduction processes that the heat propagation speed is infinite since the heat conduction equation based on Fourier's law is parabolic. This physical drawback has attracted many attempts to improve Fourier's model. Cattaneo, ${ }^{1}$ as a pioneer, and subsequently Vernotte ${ }^{2}$ and Morse and Feshbach developed a new heat conduction model, often called the $\mathrm{CV}$ equation, to replace Fourier's law. The CV equation is hyperbolic due to an additional term including the derivative of the heat flux with respect to time, which makes the heat propagation speed finite. Later, Gurtin and Pipkin ${ }^{4}$, Coleman et al. ${ }^{5}$, and Tzou ${ }^{6}$ deduced more

Key Laboratory for Thermal Science and Power Engineering of Ministry of Education, Department of Engineering Mechanics, Tsinghua University, Beijing 100084, China

*E-mail:demgzy@tsinghua.edu.cn general heat conduction equations, which were similar to the $\mathrm{CV}$ equation. The earlier hyperbolic conduction models were reviewed by Joseph and Preziosi.

The non-Fourier heat conduction effect, i.e. the finite heat propagation speed, should be taken into account for ultrafast heating or ultralow temperatures. For example, Tsai and MacDonald ${ }^{8}$ simulated heat impulsion propagation in a solid using a molecular dynamics analysis to include the finite heat conduction speed. Tzou $^{6,9,10}$ deduced various expressions for the non-Fourier heat conduction equation. $\mathrm{Xu}$ and Guo ${ }^{11}$ studied the phenomena of thermal waves in electronic chips. Brorson et al. ${ }^{12}$ measured the time for a heat pulse to penetrate a metallic film with a measured heat propagation speed of about $10^{6} \mathrm{~m} / \mathrm{s}$.

The non-Fourier phenomena was considered only for transient heat conduction conditions in these studies because the non-Fourier conduction models degenerate into Fourier's model for steady state cases. However, in recent years, the applicability of Fourier's heat conduction law has been questioned even for steady state conditions. Lepri et $a l^{13}$ numerically studied heat transport in a nonlinear onedimensional harmonic-vibrator chain and found that its thermal conductivity was approximately proportional to the square root of the particle number (chain length), which indicates the breakdown of Fourier's law. Narayan and Ramaswamy ${ }^{14}$ calculated the thermal conductivity of a one-dimensional fluid based on a momentum conservation analysis to show that the thermal conductivity is linearly related to the cube root of the system length. Maruyama ${ }^{15}$ computed the thermal conductivity of single walled carbon nanotubes (CNTs) with lengths of 6-404 nm using molecular dynamics simulations and found that the thermal conductivity increased with increasing CNT length. Such non-Fourier phenomena were attributed to the effect of the low dimensionality of the 
materials by several pioneering researchers ${ }^{16-18}$. In all these calculations, however, the obtained thermal conductivity was based on Fourier's law that is the ratio of the heat flux to the temperature gradient. Hence, this method to estimate the thermal conductivity is inappropriate when Fourier's law breaks down.

Unlike the existing solutions for non-Fourier phenomena which are basically modifications that add one or more terms, this paper shows the energy-mass duality of heat through revisiting the macroscopic nature of heat which leads to a new heat conduction law which can be applied to nanoscale heat transfer and heat transfer optimization .

\section{Dual nature of heat}

\subsection{Thermomass}

After the historic dispute on the nature of heat in the $18^{\text {th }}$ century as to whether heat is a Caloric fluid or the kinetic energy of the molecules, heat is now known to be a form of energy. However, heat is conserved during transport processes (without conversion of heat to other form of energy) which differs from mechanical or electrical energy which are dissipated, rather than conserved, during mass or charge transport processes. This implies that heat acts like a mass in mechanics or like a charge in electrical systems during heat transfer processes, that is, heat has the nature of mass.

According to Einstein's special theory of relativity ${ }^{19}$, the mass and energy of an object are related by

$$
E=M c^{2}=\frac{M_{0} c^{2}}{\sqrt{1-u^{2} / c^{2}}} \approx M_{0} c^{2}+\frac{1}{2} M_{0} u^{2}
$$

in which $M$ is the moving mass or the relativistic mass of the object, $M_{0}$ is the rest mass, $u$ is the velocity, $c$ is the speed of light in a vacuum, $E$ is the total energy or the relativistic energy of the object, and $M_{0} \mathrm{c}^{2}$ is the energy of the rest object. The relativistic energy is equal to the sum of the rest energy and the kinetic energy of the rest mass and the relativistic mass is equal to the sum of the rest mass and its equivalent mass when the velocity of the object is much less than the speed of light. Now consider heat conduction in dielectric solids with an object with rest mass, $M_{0}$, and temperature, $T . M_{0}$ is the sum of all the atomic rest masses in the object. The thermal vibration energy (i.e. phonons, which are energy quanta) is assumed to be $E_{\mathrm{D} 0}$ and the atomic relativistic mass will then be larger than the rest mass. Since the velocities of the lattice vibrations are much less than the speed of light, the increased mass due to the thermal vibrations is approximately

$$
M_{h}=\frac{E_{D 0}}{c^{2}}
$$

Thus, $M_{h}$ was called the THERMOMASS, i.e. the thermal vibration mass or the equivalent mass of the phonon gas in dielectric solids by Guo et al. ${ }^{20-24}$. This is the common view of the physical science community $^{25}$, as Feynman pointed out in his physics lecture that a hot gas is heavier than a cold gas ${ }^{26}$. Thus, the total mass of the solid is equal to the sum of its rest mass and the equivalent mass of the phonon gas. Note that the concept of "thermomass" differs from that of "caloric" in the 18th century. The thermomass is the equivalent mass of thermal energy, whereas the caloric was an imaginary, invisible, massless fluid.

\subsection{Thermomass gas}

Similar to an ordinary gas consisting of a large number of randomly moving atoms or molecules, a thermomass gas consists of a large number of randomly moving particles with relativistic masses. The thermomass gas in dielectric solids is the phonon gas, because the moving particles with relativistic masses are the phonons, while the thermomass gas in ordinary gases or metals is the thermon gas, because the moving particles with relativistic masses are the thermon ${ }^{27}$. Thus, the heat flux is essentially the directional flow of the thermomass gas due to a given temperature gradient. Therefore, these phenomena all show that heat has a dual nature, that is, heat acts like energy during its conversion with other forms of energy and heat acts like a mass during its motion. In other words, the essence of heat is its energy-mass duality.

\section{Dynamics of a thermomass gas}

Since the heat transport in dielectric solids, just like in porous media, is actually the motion of the phonon gas with the nature of mass, the heat transfer can be described by Newton's law of motion, so that a phonon gas with no net force acting on the gas molecules moves with constant mass velocity and accelerates if a net external force acts on it. The driving force here has the unit of Newton (dimension of force), which is quite different from the generalized force in irreversible thermodynamics $\mathrm{s}^{28-30}$.

\subsection{State equation of a thermomass gas ${ }^{21}$}

The Debye state equation for solids is

$$
p=-\frac{\partial E}{\partial V}+\frac{\gamma \partial E_{D 0}}{V}
$$

in which $p$ is the pressure, $V$ is the volume, and $\gamma$ is the Grüneisen constant. The first term on the right side of Eq. (3), which represents the interactions between atoms, is a negative attractive force. The second term due to the lattice vibrations is a positive repulsive force. Since the second term arises from thermal vibrations, it is the phonon gas pressure and can be also called the thermal pressure. The phonon gas pressure can be related to the energy by the state equation of a phonon gas as

$$
\frac{\gamma \partial E_{D 0}}{V}=p_{h}=\gamma \rho_{h} C T=\frac{\gamma \rho}{c^{2}}(C T)^{2}
$$

in which $\rho_{\mathrm{h}}=C T / c^{2}$ is the mass density of the phonon gas. The phonon gas pressure is proportional to the square of the temperature since the phonon gas mass is proportional to the temperature.

Just as the pressure gradient is the driving force for fluid flow, the driving force with the unit of Newton for the phonon gas motion in dielectric solids is the pressure gradient in the phonon gas. For a one-dimensional case, the pressure gradient on the phonon gas can be written as

$$
\frac{d p_{h}}{d x}=\frac{\gamma \rho C^{2}}{c^{2}} \frac{d(T)^{2}}{d x}=\frac{\gamma \rho C^{2}}{c^{2}} T \frac{d T}{d x}
$$

Therefore, the driving force for the phonon gas motion is proportional to the gradient of the square of the temperature.

\subsection{Drift velocity of a thermomass gas}

When a temperature gradient occurs in a solid, thermal energy flows from the hot to the cold regions. The thermal energy motion (transport) is usually described by the heat flux $q$. The heat flux can also be described by its velocity defined by an advection transport term as

$$
q=\rho C T u_{h}
$$


Since the thermal energy in a solid is equal to the energy of the phonon gas, $C T$ is actually the energy of the phonon gas per unit volume. Thus, $u_{\mathrm{h}}$ is the velocity of the thermal energy motion, which is equivalent to the mean phonon drift velocity or the macroscopic velocity of the phonon gas. Dividing both sides by the square of the speed of light yields

$$
\dot{m}_{h}=\frac{q}{c^{2}}=\frac{\rho C T}{c^{2}} u_{h}=\rho_{h} u_{h}
$$

in which $\dot{m}_{h}$ is the equivalent mass of the phonon gas flowing across a unit area per unit time, which is equal to the product of the mass density and the phonon gas velocity. $\dot{m}_{h}$ can be referred to as the mass velocity of phonon gas, since it is very similar to the fluid mass velocity in fluid mechanics.

\subsection{Governing equations for the motion of a thermomass gas}

The motion of an object at speeds much lower than the speed of light can be characterized by the classical Newton's laws. This is introduced in detail in textbooks on relativity ${ }^{25}$. Fluid mechanics should then be used to investigate the motion of the phonon gas once the concept of a relativistic mass is adopted and, since the drift velocity of a phonon gas is normally much less than the speed of light, Newton's law can be applied.

\subsubsection{Continuity equation}

For heat transport in a solid without an internal heat source, the equivalent mass of the phonon gas remains constant during the motion of the phonon gas. The continuity equation is then

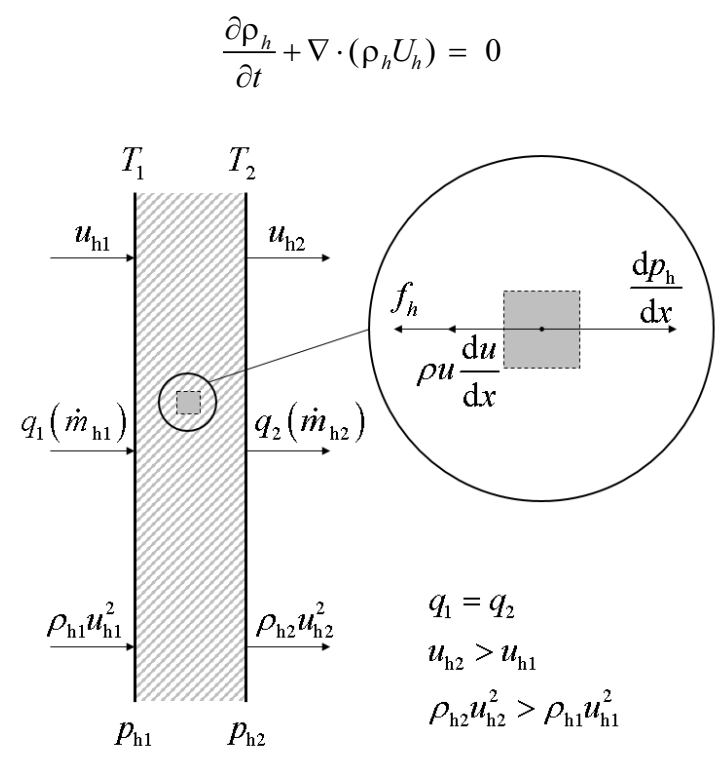

Fig.1 Schematic diagram of the thermal conduction across an infinite plate ${ }^{22}$.

For an infinite plate with different temperatures on its two sides as shown in Fig. $1\left(T_{1}>T_{2}\right)$, the continuity equation for one dimensional, steady heat conduction can be simplified as $\rho_{h} u_{h}=\dot{m}_{h}=$ const This means that the phonon gas (thermomass gas) flowing into the plate is equal to that flowing out of the plate. However, because the mass density of the phonon gas in a solid varies with temperature, the phonon gas accelerates along the flow direction such that

$$
\frac{u_{h 2}}{u_{h 1}}=\frac{\rho_{h 1}}{\rho_{h 2}}=\frac{T_{1}}{T_{2}}>1
$$

Thus, the phonon gas has an inertial force even for one dimensional steady heat conduction problems.

\subsubsection{Momentum equation and general heat conduction law}

Just as the pressure gradient is the driving force for ordinary gas flow, the driving force for phonon gas flow in solids is the pressure gradient of the phonon gas or the thermomass gas. The momentum variation in the phonon gas results in a change of the inertia. The momentum here refers to the macroscopic directional momentum carried by the macroscopic motion of the phonon gas, which differs from the apparent momentum of a phonon defined in solid physics. In addition, a resistance must exist because of the non-linearity of the lattice vibrations and defects in the solid. Thus, the equation of motion for the phonon gas can be written as in fluid mechanics as:

$$
\rho_{h} \frac{d U_{h}}{d t}+\nabla P_{h}+f_{h}=0
$$

Here, the first term represents the inertial force of the phonon gas, the second term is the pressure gradient and the third term is the resistance.

For a one dimensional steady case, Eq. (10) can be simplified to

$$
\rho_{h} u_{h} \frac{d u_{h}}{d t}+\frac{d p_{h}}{d x}+f_{h}=0
$$

this equation can be rewritten as a relationship between the temperature and the heat flux, which is the same as the general heat conduction equation $^{22}$. The equation for one-dimensional heat conduction in dielectrics is then:

$$
\tau_{\mathrm{TM}} \frac{\partial q}{\partial t}-l \rho C \frac{\partial T}{\partial t}+l \frac{\partial q}{\partial x}-b k \frac{\partial T}{\partial x}+k \frac{\partial T}{\partial x}+q=0
$$

in which

$$
\begin{gathered}
\tau_{\mathrm{TM}}=\frac{k}{2 \gamma \rho C^{2} T} \\
l=\frac{q k}{2 \gamma C(\rho C T)^{2}}=u_{\mathrm{h}} \tau_{\mathrm{TM}} \\
b=\frac{q^{2}}{2 \gamma \rho^{2} C^{3} T^{3}}
\end{gathered}
$$

Table 1. General heat conduction law reducing to other models.

\begin{tabular}{ccc}
\hline Simplified conditions & Heat condu ction models & Expression \\
\hline All inertia neglected & Fourier's law & $q=-k \nabla T$ \\
Spatial inertia neglected & Cattaneo-Vernotte model & $q+\tau \frac{\partial q}{\partial t}=-k \nabla T$ \\
Temporal inertia neglected & Steady-state non-Fourier model & $q=-(1-b) k \nabla T$ \\
\hline
\end{tabular}

The quantities $\tau_{\mathrm{TM}}$ and $l$ have dimensions of time and length, respectively, while $b$ is a dimensionless number less than unity, which is the ratio of the inertia force to the driving force. The first four terms on the left side of Eq. (12) result from the inertial effects, the fifth term represents the effect from the pressure gradient (driving force), and the sixth term results from the resistance as the 
phonon gas flows through the lattices. Eq. (12), which was developed from the concept of the mass nature of heat, describes the general relation between the temperature gradient and the heat flux vector, and is referred to as the general heat conduction law. The general heat conduction law can be simplified to the various heat conduction models with appropriate simplifications as listed in Table 1.

Eq. (12) gives a gas dynamics equation for heat conduction in a porous medium without any influence from the boundary. However, the boundary effects become important in nanosystems and a Brinkman term was introduced into the traditional form of Darcy's law when transitional flow between boundaries should be taken into account $^{31}$. In analogy with this extension for flows in porous hydrodynamics, an additional term should be included in the general heat conduction law, Eq. (12)

$$
\tau_{\mathrm{TM}} \frac{\partial \mathbf{q}}{\partial t}+2 l \nabla \mathbf{q}-b \kappa \nabla T+\kappa \nabla T+\mathbf{q}-\mu \nabla^{2} \mathbf{q}=0
$$

where $\mu$ is the effective viscosity of the thermomass gas. In porous media, the Brinkman extension predicts a boundary layer with the nonslip or slip boundary condition significantly changing the drift velocity. However this boundary layer is usually very thin and can be ignored in large systems. Similarly, the Brinkman term in Eq. (16) reflects the additional drag due to the walls in the system and should be considered in nanoscale systems when the characteristic system length is comparable with the friction boundary layer of the thermomass gas. In other words, the Brinkman extension is necessary only if the system Knudsen number is large. This extension has also been suggested by Cimmelli et al. ${ }^{32-34}$ in an interesting example similar to the nonlinear extension of the GuyerKrumhansl (GK) equation where the effective viscosity is related to the square of the mean free path of the energy carriers.

\section{Boltzmann transport equation of a thermomass gas}

The phonon hydrodynamic equations can be derived from the phonon Boltzmann equation ${ }^{24,35-39}$. Generally, the objective is to find the real phonon distribution function, $f$, and then the governing equations. However, many assumptions are required to solve the Boltzmann equation as in fluid mechanics. Different approaches for dealing with the Boltzmann equation then lead to different governing equations. In the following section, the Boltzmann equation will be analyzed with the thermomass concept with the results compared with other solutions for phonon hydrodynamics. This will show the inherent similarity between the governing equations for heat conduction based on thermomass theory and that based on phonon hydrodynamics.

The macroscopic variables, such as the internal energy density, $E$, and the heat flux, $q$, are related to the microscopic distribution function as

$$
\begin{aligned}
& E=\sum_{s} \int_{\boldsymbol{k}} \hbar \omega_{s} f_{s} \\
& q_{i}=\sum_{s} \int_{\boldsymbol{k}} \hbar \omega_{s} \frac{\partial \omega_{s}}{\partial k_{i}} f_{s}
\end{aligned}
$$

where $s$ is the index of the phonon branches, $k$ is the wave vector, and $\omega_{s}$ is the frequency. The integral is over the whole $k$ space and then summed over all branches. Sussmann \& Thellung ${ }^{39}$ deduced a heat conduction equation by neglecting the Umklapp processes in perfect dielectric crystals using a mean free time approximation on the distribution function. Guyer \& Krumhansl $^{36}$ used an eigenvalue analysis of the Boltzmann equation to obtain the governing equation known as the Guyer-Krumhansl equation

$$
\tau_{R} \frac{\partial \mathbf{q}}{\partial t}+\mathbf{q}=-\kappa \nabla T+l^{2}\left(\nabla^{2} \mathbf{q}+2 \nabla \nabla \cdot \mathbf{q}\right)
$$

where $l$ is the mean free path of the phonons. The form of Eq. (19) is similar to the results of Sussmann \& Thellung ${ }^{39}$ and was further analyzed by Hardy \& Albers ${ }^{37}$. The impact on the heat conduction of Umklapp scattering and other quasi-momentum non-conserving processes in Eq. (19) is described by a relaxation time, $\tau_{\mathrm{R}}$.

The distribution function for the equilibrium state follows the Planck distribution,

$$
f_{E}=\frac{1}{\exp \left(\hbar \omega / k_{B} T\right)-1}
$$

The normal process tends to relax the distribution function to the displaced Planck distribution

$$
f_{D}=\frac{1}{\exp \left(\hbar \omega-\hbar \boldsymbol{k} \cdot \boldsymbol{u} / k_{B} T\right)-1}
$$

where $\boldsymbol{u}$ is the so-called drift velocity of the phonon gas. The resistive quasi-momentum non-conserving process tends to relax the distribution function to the equilibrium Planck distribution $f_{\mathrm{E}}$. Thus, a relaxation type of phonon Boltzmann equation can be written as ${ }^{35,38}$

$$
\left(\frac{\partial}{\partial t}+\boldsymbol{v}^{s} \cdot \nabla\right) f^{s}=\frac{f_{E}^{s}-f^{s}}{\tau_{R}}+\frac{f_{D}^{s}-f^{s}}{\tau_{N}}
$$

where $v^{s}$ is the group velocity and $\tau_{\mathrm{N}}$ is the relaxation time of the normal processes.

In low temperature prefect crystals, the normal processes are dominant and the Umklapp processes are rare, so $\tau_{\mathrm{N}}<<\tau_{\mathrm{R}}$. Then, $f_{\mathrm{D}}$ is a good approximation to the real distribution ${ }^{39}$. This is the simplest assumption to demonstrate the structure of the solution of the Boltzmann equation. The deviation from $f_{\mathrm{D}}$ can be taken into account by a Chapman-Enskog expansion.

Substituting $f_{\mathrm{D}}$ into Eq. (22) gives

$$
\left(\frac{\partial}{\partial t}+\boldsymbol{v}^{s} \cdot \nabla\right) f_{D}^{s}=\frac{f_{E}^{s}-f_{D}^{s}}{\tau_{R}}
$$

In the transport theory for gases ${ }^{40}$, multiplying the Boltzmann equation by the momentum of the molecules, $m v$, gives the momentum conservation equation. In phonon gases, $\hbar \omega$ is the energy of a phonon and $\hbar \omega / c^{2}$ is the equivalent mass according to thermomass theory. In this way, $\hbar \omega v_{\mathrm{i}} / c^{2}$ represents the momentum of a phonon. This is the real momentum and differs from the quasimomentum of phonons, i.e. $\hbar k$. Multiplying Eq. (23) by $\hbar \omega / c^{2}$ and $\hbar \omega v_{\mathrm{i}} / c^{2}$ leads to the mass and momentum conservation equation for phonon gases as in hydrodynamics. However, unlike ideal gases in channels, the phonon gases experience a resistance from the Umklapp processes or crystal defects. This difference is reflected by a momentum sink term with the collision term in Eq. (23) multiplied by $\hbar \omega v_{\mathrm{i}} / c^{2}$. In practice, the parameter $c^{2}$ can be cancelled out from the equations since it is a constant.

Multiplying Eq. (23) by $\hbar \omega / c^{2}$ and $\hbar \omega v_{\mathrm{i}} / c^{2}$ and integrating in $k$ space yields 


$$
\begin{gathered}
\frac{\partial \int_{k} f_{D}^{s} \hbar \omega}{\partial t}+\int_{k} v^{s} \cdot \nabla f_{D}^{s} \hbar \omega=\frac{\int_{k}\left(f_{E}^{s}-f_{D}^{s}\right) \hbar \omega}{\tau_{\mathrm{R}}} \\
\frac{\partial \int_{k} f_{D}^{s} \hbar \omega v_{i}}{\partial t}+\int_{k} \boldsymbol{v}^{s} \cdot \nabla f_{D}^{s} \hbar \omega v_{i}=\frac{\int_{k}\left(f_{E}^{s}-f_{D}^{s}\right) \hbar \omega v_{i}}{\tau_{\mathrm{R}}}
\end{gathered}
$$

When the drift velocity, $\boldsymbol{u}$, is small, a Taylor expansion of $f_{\mathrm{D}}$ around equilibrium up to the second order gives

$$
\begin{aligned}
f_{D} & \left.=f_{E}+\left.\frac{\partial f_{D}}{\partial \boldsymbol{u}}\right|_{\Delta u=0} \Delta \boldsymbol{u}+\left.\frac{1}{2} \frac{\partial^{2} f_{D}}{\partial \boldsymbol{u}^{2}}\right|_{\Delta u=0}(\Delta \boldsymbol{u})^{2}+o(\Delta \boldsymbol{u})^{2}\right) \\
& =f_{E}+\frac{\partial f_{E}}{\partial \omega}(\boldsymbol{k} \cdot \boldsymbol{u})+\frac{\partial^{2} f_{E}}{\partial \omega^{2}}(\boldsymbol{k} \cdot \boldsymbol{u})^{2}+o\left((\Delta \boldsymbol{u})^{2}\right) \\
& =f_{E}+f_{+}+f_{++}+o\left((\Delta \boldsymbol{u})^{2}\right)
\end{aligned}
$$

where $f_{\mathrm{E}}$ and $f_{++}$are even functions in $k$ space and $f_{+}$is odd. Substituting the second order expansion of $f_{\mathrm{D}}$ into Eq. (24) gives the energy balance relation

$$
\frac{\partial E}{\partial t}+\nabla_{j} q_{j}=0
$$

Substituting the second order expansion of $f_{\mathrm{D}}$ into Eq. (25) gives the governing equation

$$
\frac{\partial q_{i}}{\partial t}+\frac{15}{16} \nabla_{j} \frac{q_{i} q_{j}}{E}+\frac{1}{3} \nabla_{j} \int_{k} f_{E}^{s} \hbar \omega\left(v^{s}\right)^{2}=-\frac{q_{i}}{\tau_{\mathrm{R}}}
$$

The third term on the left side of Eq. (28) assumes a cubic symmetry condition.

The momentum transport equation, Eq. (28), can be compared with that in thermomass theory (Eq. 12):

$$
\frac{\partial q_{i}}{\partial t}+\nabla_{j} \frac{q_{i} q_{j}}{E}+\nabla_{i} p_{\mathrm{h}}=-\beta \frac{q_{i}}{E}
$$

Eq. (29) has been modified by adding the thermomass conservation relation, Eq. (8), to create a form that is a parallel with Eq. (28). The isotropic thermomass pressure is represented in the phonon Boltzmann method as

$$
p_{\mathrm{h}}=\frac{1}{3} \int_{\boldsymbol{k}} f_{E}^{s} \frac{\hbar \omega}{c^{2}}\left(v^{s}\right)^{2}=\iint_{ \pm \pi / a} f_{E}^{s}(\boldsymbol{x}, t, \boldsymbol{k}) \frac{\hbar \omega}{c^{2}} v_{x}^{2} d k_{x} d k_{y} d k_{z}
$$

The temperature gradient driving the heat flux corresponds to the pressure difference of the heat carriers as in hydrodynamics. The phonon gas pressure can be obtained either macroscopically by Eq. (4) or microscopically by Eq. (30). The predicted relaxation time for $\mathrm{Si}$ at $300 \mathrm{~K}$ based on the first method is $1.4 \times 10^{-10} \mathrm{~s}$ for $k_{\text {bulk }}=149 \mathrm{~W}$ $\mathrm{m}^{-1} \mathrm{~K}^{-1}, C_{\mathrm{v}}=704.6 \mathrm{~J} \mathrm{~kg}^{-1} \mathrm{~K}^{-1}, \rho=2330 \mathrm{~kg} \mathrm{~m}^{-3}$, and $\gamma=1.5^{41}$. The second method predicts the thermal relaxation time to be $0.5 \times 10^{-10} \mathrm{~s}$ ${ }^{42}$. The experimental value ${ }^{42}$ is about $1.5 \times 10^{-10} \mathrm{~s}$. Thus, both methods give the same order-of-magnitude as the measured value.

The three terms on the left side of Eq. (28) come from $f_{+}, f_{++}$and $f_{\mathrm{E}}$. If only the third term is retained, Eq. (30) reduces to the traditional Fourier's law of heat conduction. If the terms from $f_{\mathrm{E}}$ and $f_{+}$are retained, Eq. (30) reduces to the telegraphic Cattaneo-Vernotte thermal wave equation. The displaced Planck distribution, when expanded to the second order, gives a governing equation derived from the Boltzmann equation that is similar to thermomass theory. However, the coefficient of the convective term is $15 / 16$ in Eq. (28) but unity in Eq. (29). This is related to the change in the phonon energy caused by the Doppler effect. The phonon gas differs from a gas consisting of real particles since the phonon energy varies with the drift motion, so the convective term is "gibbous".

\section{Essence of Fourier's heat conduction law}

\subsection{Fluid flow in porous media: Darcy's law}

The fundamental equation characterizing fluid flow in porous media is Darcy's law

$$
\dot{m}=\rho u_{m}=-K_{m} \frac{d p}{d x}
$$

in which $\dot{m}$ is the mass flux and $K_{\mathrm{m}}$ is the permeability of the porous media. Therefore, the macroscopic fluid velocity in a porous media is proportional to the driving force acting on the fluid (the pressure gradient).

Eq. (31) can be rewritten as

$$
\begin{gathered}
-\frac{d p}{d x}=\frac{\rho}{K_{m}} u_{m}=f_{m o} \\
f_{m o}=\beta_{m p} u_{m}
\end{gathered}
$$

in which $f_{\mathrm{mo}}$ is the viscosity-induced resistant force per unit volume, $\beta_{\mathrm{mo}}=\rho / K_{\mathrm{m}}$. Eq. (32) represents the balance between the driving force and the resistant force with the inertial force acting on the fluid being ignored. Since the resistance force is linearly related to the velocity, the velocity in a porous media is also proportional to the driving force. However, when the fluid velocity or the Reynolds number (Re) exceeds a critical value ( $\operatorname{Re}>10)$, Darcy's law breaks down because the inertial force in the fluid cannot be neglected relative to the other terms.

\subsection{Heat flow in porous media: Fourier's law}

The heat flux can be related to the mass flow rate of the thermomass by introducing the concept of a thermomass fluid. Putting the relationship between the heat flow and the velocity of the phonon gas into Fourier's model gives

$$
\rho_{h} u_{h}=-\frac{K d T}{c^{2}} \frac{d}{x}
$$

Writing this in terms of the pressure gradient in the phonon gas gives:

$$
\begin{gathered}
-\frac{d p_{h}}{d x}=\left(\frac{2 \gamma C \rho_{h}^{2}}{K}\right) u_{h}=f_{h} \\
f_{h}=\beta_{h} u_{h}
\end{gathered}
$$

Eq. (35) is the same as Eq. (32). As Eq. (32) is for flow in porous media, Eq. (35) is the balance equation between the driving force per unit volume, $\mathrm{d} p_{\mathrm{h}} / \mathrm{d} x$, and the resistance force per unit volume, $f_{\mathrm{h}}$, induced by the phonon scattering. Eq. (36) indicates that the resistance force is proportional to the phonon gas velocity with a proportionality constant.

Therefore, the physical essence of Fourier's law of heat conduction is that the driving force (or the pressure gradient) of the phonon gas flow is in equilibrium with its resistance force. In other words, Fourier's law characterizes the heat motion as the pressure gradient balanced by the resistance. 


\section{Applications of the general heat conduction law 6.1 Steady non-Fourier heat conduction in carbon nanotubes}

Thermal conduction processes that are not described by Fourier's model are referred to as non-Fourier phenomena. When the inertial force can be ignored and the resistance is linearly related to the velocity, Fourier's conduction law may then be used to calculate the resistance in the equation of motion, Eq. (12). For one-dimensional, steady state heat conduction, the equation of motion, Eq. (12), can be written as:

$$
\rho_{h} u_{h} \frac{d u_{h}}{d x}+2 \gamma \rho_{h} C \frac{d T}{d x}+\frac{2 c^{2} \gamma \rho_{h}^{2} C}{k} u_{h}=0
$$

Using the continuity equation, Eq. (37) can be rewritten as ${ }^{22}$

$$
\left(1-\frac{q^{2}}{\rho^{2} C^{3} T^{3}}\right) K \frac{d T}{d x}+q=0
$$

This can be regarded as a generalized form of Fourier's law for steady heat conduction, but is actually a first order approximation of Eq. (12). Eq. (38) shows that the heat flux is not just proportional to the temperature gradient with higher heat fluxes resulting in deviations from Fourier's law.

In these cases, the thermal conductivity in nanomaterials calculated based on experimental data for the heat flux and the temperature gradient is not the intrinsic thermal conductivity of the material but a quantity related to the intrinsic thermal conductivity, which will be referred to as the apparent thermal conductivity

$$
-\frac{q}{(d T / d x)}=K_{D}=K\left(1-\frac{q^{2}}{\rho^{2} C^{3} T^{3}}\right) \quad, \quad K_{D}>K
$$

where $K$ is the intrinsic thermal conductivity and $K_{\mathrm{D}}$ is the apparent thermal conductivity. Therefore, the apparent thermal conductivity extracted from the experimental data based on Fourier's law of heat conduction is actually a function of the heat flux, which is always less than the intrinsic thermal conductivity.

Now consider one dimensional, steady heat conduction in carbon nanotubes with lengths of $40 \mu \mathrm{m}, 80 \mu \mathrm{m}$ and $120 \mu \mathrm{m}$. The boundary conditions on both ends are constant temperatures with a temperature difference $\Delta T=T_{1}-T_{2}=20 \mathrm{~K}$. The temperature independent thermal conductivity of the carbon nanotube, $K$, is $K=$ $5000 \mathrm{~W} / \mathrm{m} \mathrm{K}$. If the temperature difference remains constant, varying the carbon nanotube length is equivalent to changing the heat flux. When the heat flux is very large, the inertial force of the phonon gas cannot be neglected, which leads to nonlinear temperature profiles along the nanotube as shown in Fig. 2. When the nanotube is longer, the heat flux is less, and the temperature profiles are closer to linear. Fig. 3 shows the apparent thermal conductivities of carbon nanotubes with various lengths (heat fluxes), where the apparent thermal conductivities, $K_{\mathrm{D}}$, are always less than the intrinsic thermal conductivity, $K$. As the nanotube length increases, the heat flux decreases and $K_{\mathrm{D}}$ approaches $K$. For $q^{2} / \rho^{2} C^{3} T^{3}<<1$ in Eq. (38), Fourier's law is then applicable and the temperature profiles are linear. If the experimentally measured thermal conductivity is assumed to be the intrinsic thermal conductivity rather than the apparent thermal conductivity, the thermal conductivity would be mistakenly found to vary with the nanotube length (see Fig. 3). The heat fluxes through carbon nanotubes can reach $10^{12} \mathrm{~W} / \mathrm{m}^{2}{ }^{15}$. The term $q^{2} / \rho^{2} C^{3} T^{3}$ is then not much less than unity, which may be one of the factors for the variation of the thermal conductivity with the carbon nanotube length in addition to the ballistic transport of phonons in Maruyama's ${ }^{15}$ calculated results.

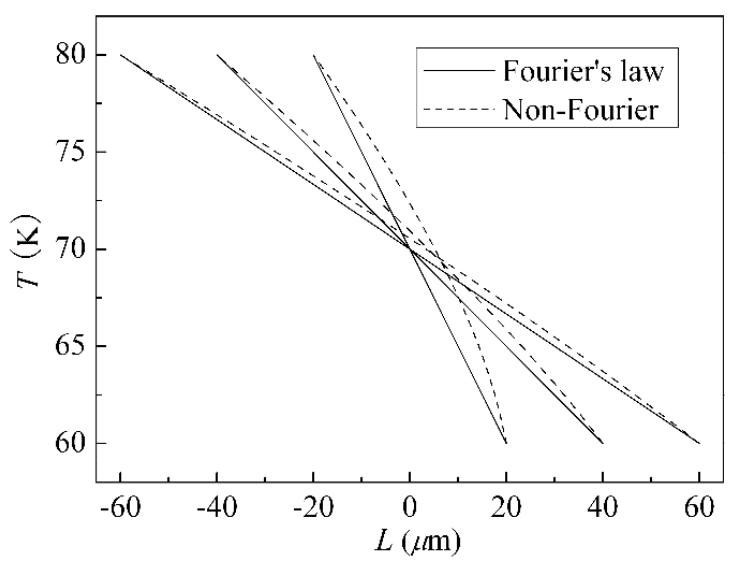

Fig.2 Temperature profiles along a carbon nanotube ${ }^{22}$.

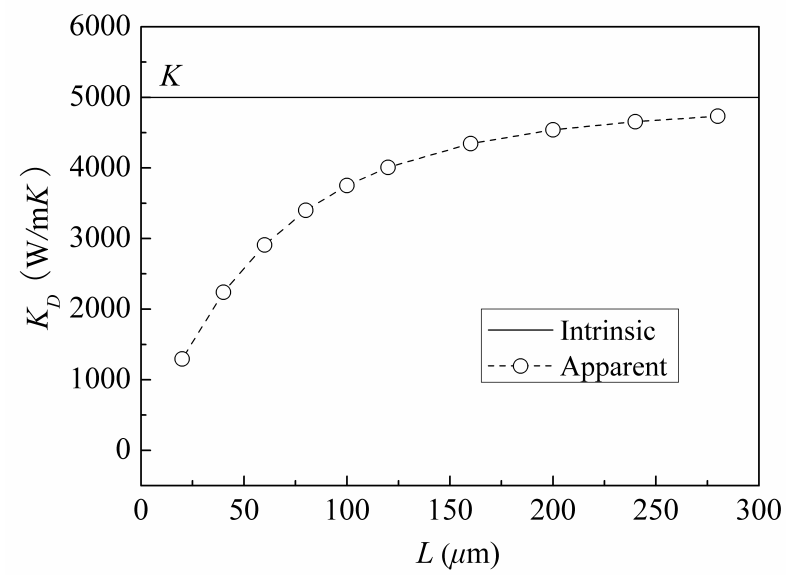

Fig. 3 Dependence of the apparent thermal conductivity on carbon nanotube length ${ }^{22}$

\subsection{Effective Thermal Conductivity of Nanostructures}

The idea that phonons act like gases can be traced back to the $1920 \mathrm{~s}$ in a theory proposed by Debye et $a l^{43}$ They predicted the thermal conductivity in terms of MFP as in the kinetic theory of gases. Guyer and Krumhansl solved the phonon Boltzmann equation using a linear assumption and obtained a transport model (GK model) containing transient and nonlocal terms. For steady heat conduction in straight wires or films, the GK model can be simplified as ${ }^{36}$

$$
\kappa \nabla T=-\boldsymbol{q}+\frac{l_{R} l_{N}}{5} \nabla^{2} \boldsymbol{q}
$$

where $l_{\mathrm{R}}=v_{\mathrm{s}} \tau_{\mathrm{R}}$ and $l_{\mathrm{N}}=v_{\mathrm{s}} \tau_{\mathrm{N}}$ are the MFPs of the resistive scattering and normal scattering, respectively. The nonlocal term suggests that the heat flux in a wire or film is non-uniform in each cross section. If $\nabla^{2} \mathrm{q}$ is negligible, Eq. (40) reduces to Fourier's law. If $\nabla^{2} \mathrm{q}$ dominates, Eq. (40) has a form like the Navier-Stokes equations and predicts a parabolic heat flux profile like the velocity profile in Poiseuille flow. A characteristic length can be defined as, $l_{G}^{2}=\kappa \nabla T / \nabla^{2} \boldsymbol{q}$ with its ratio relative to the characteristic size of the nanosystem measured by a Knudsen number, $\mathrm{Kn}_{\mathrm{G}}=l_{\mathrm{G}} / L$, where $L$ is the film thickness or the wire diameter. Note that the characteristic length, $l_{\mathrm{G}}$, is differs from 
the ordinary MFP. The latter is $l_{\mathrm{R}}$ in general. The Poiseuille flow of a phonon gas occurs when $\mathrm{Kn}_{\mathrm{G}}>>1$, i.e. the term, $l_{\mathrm{G}}^{2} \nabla^{2} \boldsymbol{q}$, is much greater than $\boldsymbol{q}$ in Eq. (40).

Darcy's law is only a simplified description of porous flow in general materials. A general constitutive equation for porous flow must include the effects of acceleration, nonlinear drag and advection. Brinkman's equation is a well-known generalization to Darcy's law,

$$
\boldsymbol{f}=-\frac{\mu}{K} \boldsymbol{u}_{\mathrm{m}}+\mu \nabla^{2} \boldsymbol{u}_{\mathrm{m}}
$$

where $\boldsymbol{f}$ is the total resistance, $\boldsymbol{u}_{\mathrm{m}}$ is the fluid velocity, $\mu$ is the fluid viscosity, and $K$ is the permeability. The first term in Eq. (41) leads to Darcy flow, so this is called the Darcy friction term. The second term in Eq. (41) is from normal fluid flow and is called the Brinkman term. The Brinkman term describes the advection effect. The characteristic length, $l_{\mathrm{B}}=K^{1 / 2}$, reflects the attenuation length of the boundary effect. Therefore, the dimensionless Brinkman number, $\mathrm{Br}$ $=l_{\mathrm{B}} / L$, reflects the importance of the boundary viscous friction compared to the Darcy friction. If $\mathrm{Br}<<1$, the boundary effect region is much smaller that the channel width and the velocity profile is nearly uniform across the cross section, which agrees with the prediction of Darcy's law. Conversely, if $\mathrm{Br}>>1$, the flow is mainly impeded by the boundary drag; thus, the velocity profile approaches that for Poiseuille flow. Since thermomass theory treats the phonon gas as a fluid with mass in the porous medium, the constitutive equation for steady heat conduction in nanosystems systems is ${ }^{44}$,

$$
-\kappa \nabla T=\boldsymbol{q}-\frac{\mu_{\mathrm{h}} \tau_{\mathrm{R}}}{\rho_{\mathrm{h}}} \nabla^{2} \boldsymbol{q}=\boldsymbol{q}-l_{B}^{2} \nabla^{2} \boldsymbol{q}
$$

An analysis of the phonon Boltzmann derivation also shows that the Brinkman term arises microscopically from the Chapman-Enskog expansion of the distribution function, which is exactly the same as the microscopic foundation of the viscous stress term in the NavierStokes (NS) equations. In this case, the heat transport is in the ballistic-diffusive regime ${ }^{45,46}$. Similarly, recent work has also indicated that a hydrodynamic description of localized electromagnetic waves is possible in complex open systems. The analytical solution to Eq. (42) was then obtained for the DarcyBrinkman flow of a phonon gas ${ }^{44}$. Solutoins can also be obtained for other geometries like nanofilms and nanowires. If $l_{B}$ is constant and the heat flux vanishes at the boundaries, the heat flux profile for fully developed flow in a nanofilm is

$$
q(r)=-\kappa \nabla T\left[1-\frac{\cosh \left(r / l_{B}\right)}{\cosh \left(L / 2 l_{B}\right)}\right]
$$

where $r[0, L / 2]$ is the distance from the center line. Then, the effective thermal conductivity is defined by the integral

$$
\kappa_{\mathrm{eff}}^{\mathrm{nf}}=-\frac{\int_{L} q d y}{\nabla T L}=\kappa_{0}[1-2 \mathrm{Brtanh}(1 / 2 \mathrm{Br})]
$$

For a nanowire, the effective thermal conductivity is

$$
\kappa_{\mathrm{eff}}^{\mathrm{nw}}=\kappa\left[1-4 \mathrm{Br} \cdot \frac{J_{1}(i / 2 \mathrm{Br})}{i J_{0}(i / 2 \mathrm{Br})}\right]=\kappa\left[1-\frac{\sum_{t=0}^{\infty} \frac{(4 \mathrm{Br})^{-2 t}}{t !(t+1) !}}{\sum_{t=0}^{\infty} \frac{(4 \mathrm{Br})^{-2 t}}{t ! t !}}\right]
$$

Illustrative solutions of the Navier-Stokes model and the DarcyBrinkman model are presented for nanofilms in Fig. 4. For comparison, we assumed $l_{\mathrm{G}}=l_{\mathrm{B}}$; thus, $\mathrm{Kn}_{\mathrm{G}}=\mathrm{Br}$. At small $\mathrm{Br}$, the Navier-Stokes model predicts a large flow rate with the maximum $q$ much larger than $q_{0}$. For the Darcy-Brinkman model, the viscous layer is constrained in the near wall region with the central flow region having a uniform heat flux, $q_{0}$. As Br increases, the profile of the Navier-Stokes model is asymptotic to the Darcy-Brinkman model. The difference between the predicted $\kappa_{\text {eff }}$ for the NavierStokes model and Darcy-Brinkman model is $9.1 \%$ at $\mathrm{Br}=1$ and $0.6 \%$ at $\mathrm{Br}=4$. Thus, neglecting the Darcy friction term could cause considerable error at moderate sizes. The predicted results for silicon nanofilms and nanowires are compared with experiment data in Fig. 5 which shows that the present model can well predict the effective thermal conductivity of both nanofilms and nanowires.
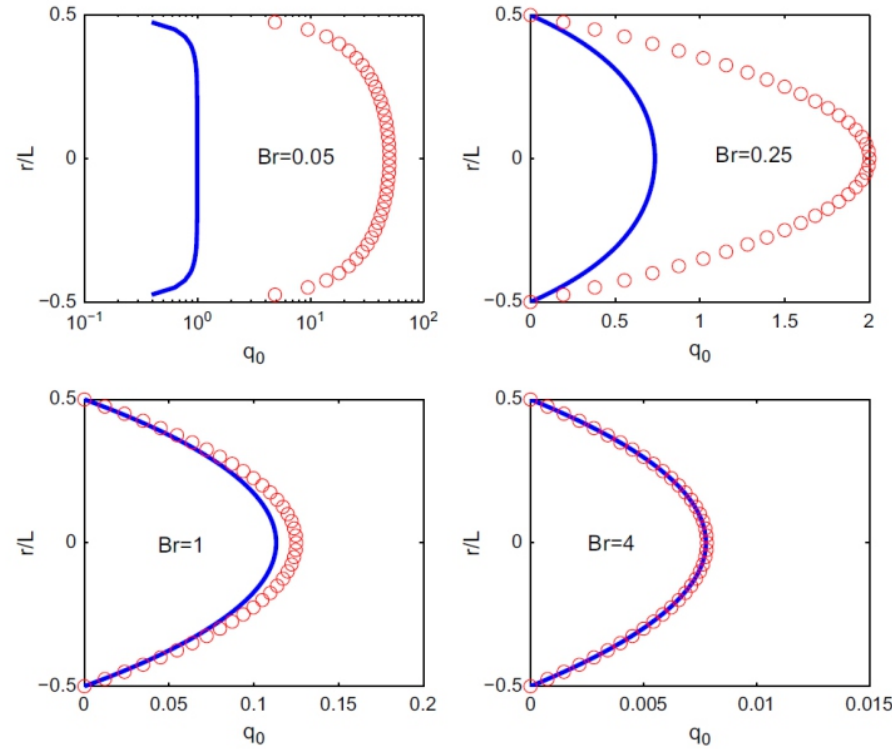

Fig. 4 Velocity profiles based on the Navier-Stokes model (circles) and the Darcy-Brinkman model (lines) for different Brinkman numbers $(\mathrm{Br})^{47}$.

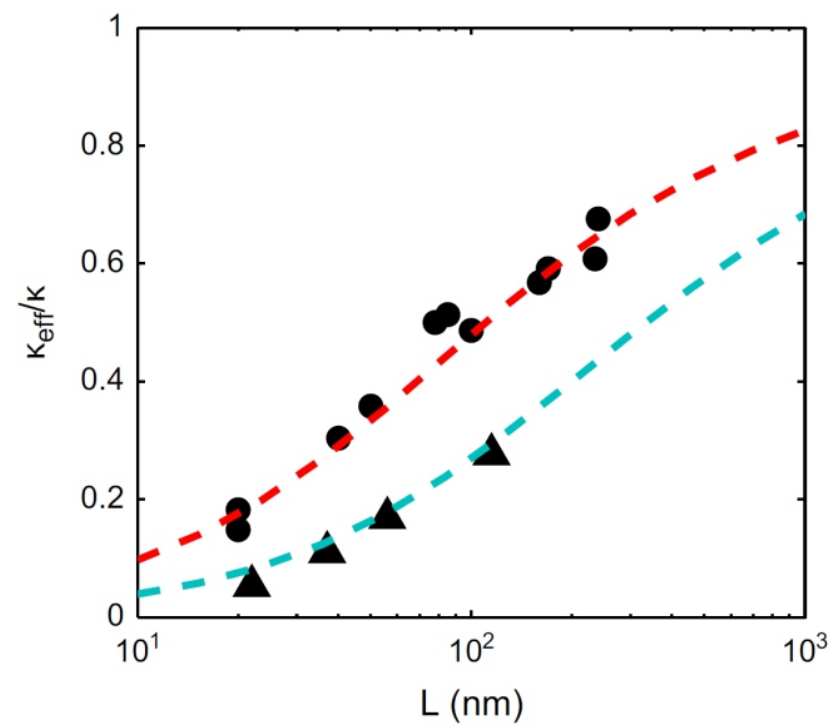

Fig. 5 The effective thermal conductivity of $\mathrm{Si}$ nanosystems predicted by the phonon gas model compared with the experimental data. Circles: experimental data for nanofilms; Triangles: experimental data for nanowires ${ }^{48}$. 


\section{Applications of thermomass energy for heat transfer optimization}

The minimum entropy generation principle has been applied to optimize the heat transfer by minimizing the entropy generation ${ }^{49,50}$. However, for a counter-flow heat exchanger, the effectiveness increases rather than decreases with increasing entropy generation when the effectiveness is less than 0.5. In addition, Shah et al. ${ }^{51}$ analyzed the irreversibility of 18 different types of heat exchanger flow arrangements and concluded that the minimum entropy generation associated with the maximum efficiency was not always applicable to heat exchanger analyses. That is, minimizing the entropy generation of a heat transfer system does not always give the best heat transfer performance, which really needs an alternative physical quantity.

\subsection{Thermomass energy and entransy}

The analogies of the transport processes for electricity, fluid mechanics and thermal science listed in Table 2 show that thermomass corresponds to the electric charge and the fluid mass, while the thermal potential corresponds to the electrical and gravitational potentials, the thermomass potential energy corresponds to the electrical and mechanic potential energies and Fourier's law corresponds to Ohm's law and Darcy's law.

Guo et $a l^{52}$ defined the quantity entransy as a simplified expression for the thermomass potential energy with the following integral form ${ }^{53}$,

$$
G=\frac{U T}{2}=\frac{1}{2} M c_{v} T^{2}
$$

where $M$ is the solid object mass, $T$ is the temperature, $c_{v}$ is the constant volume specific heat, $U$ is the internal energy of the solid object, and $G$ is termed the entransy, which is a state quantity.

\subsection{Entransy dissipation and entransy dissipation- based thermal resistance}

For a heat conduction process without internal heat sources, multiplying both sides of energy conservation equation by the temperature, $T$, yields the entransy balance equation

$$
\frac{\partial\left(\rho c T^{2} / 2\right)}{\partial t}=-\nabla \cdot(q T)+q \cdot \nabla T
$$

where the left side is the time variation of the entransy and the first and second terms on the right side are the entransy flux and the local entransy dissipation rate, $\dot{g}_{g}=q \cdot \nabla T$. The entransy balance equation shows that the entransy is not conserved due to the heat transfer irreversibility induced entransy dissipation even though the heat is conserved during a heat conduction process. For a given heat flux, minimization of the entransy dissipation leads to the minimum temperature difference. Conversely, for a given temperature difference, maximization of the entransy dissipation results in the maximum heat flux. That is, the entransy dissipation extremum corresponds to the optimal heat conduction performance.

Similarly, for steady-state convective heat transfer processes, the entransy dissipation extremum results in the best heat transfer performance with the maximum convective heat transfer coefficient for the given conditions. In addition, as with electrical conduction where the electric resistance can be expressed as the electrical energy dissipation divided by the square of the current for the whole system, the thermal resistance of a thermal system is the ratio of the entransy dissipation rate divided by the square of the heat transfer rate

$$
R_{d}=G_{d} / Q^{2}
$$

\begin{tabular}{|c|c|c|c|c|}
\hline Discipline & $\begin{array}{l}\text { Non-dissipation } \\
\text { quantity }\end{array}$ & Potential & Dissipation quantity & $\begin{array}{l}\text { Transport } \\
\text { law }\end{array}$ \\
\hline Electric & Electric charge & Electric potential & $\begin{array}{c}\text { Electric potential } \\
\text { energy }\end{array}$ & Ohm's law \\
\hline conduction & $Q_{\mathrm{ev}}$ & $V$ & $E_{e}=Q_{e v} V / 2$ & $i=-\sigma \frac{\partial v}{\partial x}$ \\
\hline Fluid mass & Mass & $\begin{array}{c}\text { Gravitational } \\
\text { potential }\end{array}$ & $\begin{array}{c}\text { Mechanic potential } \\
\text { energy }\end{array}$ & Darcy's law \\
\hline diffusion & $M$ & $H g$ & $E_{m}=M H g / 2$ & $\dot{m}=-\frac{k}{\mu} \frac{\partial p}{\partial y}$ \\
\hline Heat & Thermomass & Thermal potential & $\begin{array}{c}\text { Thermomass potential } \\
\text { energy }\end{array}$ & $\begin{array}{l}\text { Fourier's } \\
\text { law }\end{array}$ \\
\hline conduction & $M_{\mathrm{h}}=U / c^{2}[\mathrm{~kg}]$ & $C T[\mathrm{~J} / \mathrm{kg}]$ & $E_{h}=U C T / 2 c^{2}$ & $\dot{q}=-k \frac{\partial T}{\partial y}$ \\
\hline
\end{tabular}

Table 2. Analogies among electricity, fluid mechanics and heat transfer. 
where $G_{d}$ is the entransy dissipation rate over the whole heat transfer domain and $Q$ is the total heat transfer. Thus, the entransy dissipation extremum principle can be described as the principle of the minimum entransy dissipation-based thermal resistance.

\subsection{Heat conduction and convection optimization}

The minimum entransy dissipation-based thermal resistance principle has been used to optimize heat conduction, heat convection and thermal radiation processes ${ }^{53}$. For instance, the volume-point heat conduction problem shown in Fig. 6 has a uniform internal heat source in a two-dimensional region with length $L$ and width $H$. The heat is only released to the ambient from a point boundary such as the cooling surface with opening $W$ and temperature $T_{0}$ on one side. Some high thermal conductivity material (HTCM) is to be added in this region to reduce the device temperature. The HTCM distribution is to be optimized to minimize the average device temperature for a given amount of HTCM.

For example, consider an internal heat source of $100 \mathrm{~W} / \mathrm{cm}^{2}$, $L=H=5 \mathrm{~cm}, W=0.5 \mathrm{~cm}$, and a cold temperature of $10 \mathrm{~K}$. The thermal conductivity of the base material is $3 \mathrm{~W} / \mathrm{m} \mathrm{K}$, while that of the HTCM is $300 \mathrm{~W} / \mathrm{m} \mathrm{K}$. The result with a uniformly distributed HTCM is shown in Fig. 7a with an average temperature of $544.7 \mathrm{~K}$. The optimal HTCM arrangement based on the minimum entransy dissipation-based thermal resistance principle is shown in Fig. 7 b. The average temperature is $51.6 \mathrm{~K}$, a $90.5 \%$ reduction compared with the result for the uniformly distributed HTCM.

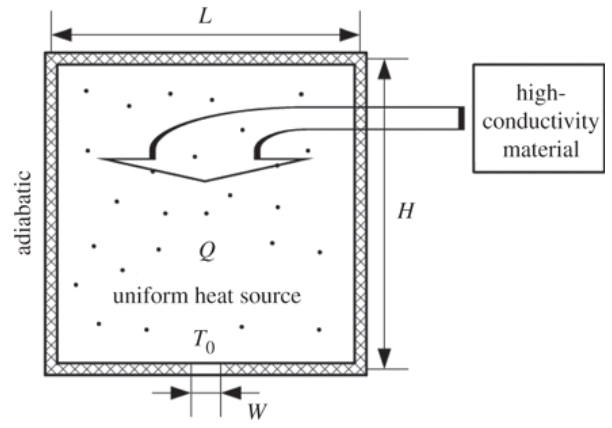

Fig. 6. Two-dimensional heat conduction with a uniformly internal heat source ${ }^{54}$.
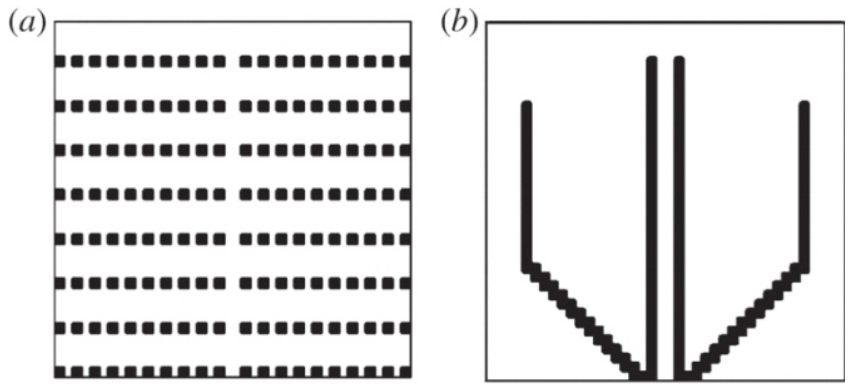

Fig. 7 Different HTCM arrangements ${ }^{54}$ : a) uniform HTCM distribution. b) HTCM distribution optimized by entransy theory.

Optimization of convective heat transfer processes using the minimum entransy dissipation-based thermal resistance principle indicates that the optimal flow pattern for laminar heat transfer in a circular tube has multiple longitudinal vortices ${ }^{55}$, while that for turbulent heat transfer between two parallel plates has several small counter-clockwise eddies near the plate ${ }^{56}$, as shown in Figs. 8 and 9.
Thus, these optimization examples indicate that the minimum entransy dissipation-based thermal resistance principle can be used to optimize convective heat transfer processes to reduce the pumping power for the given heat flux.

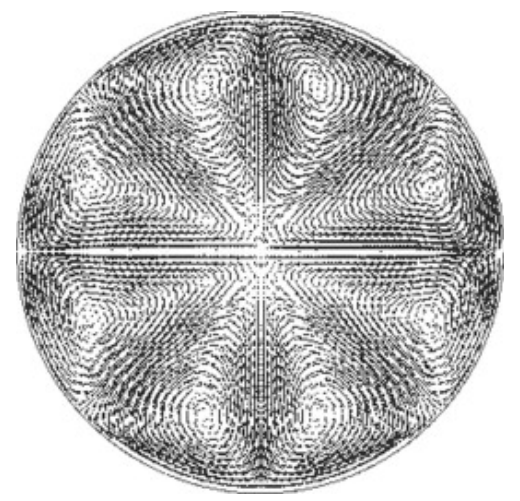

Fig. 8 Optimal flow field for laminar heat transfer in a circular tube ${ }^{55}$.

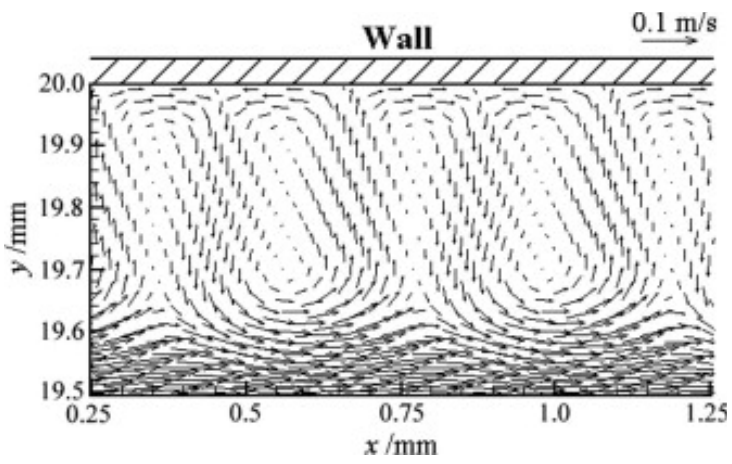

Fig. 9 Optimal velocity field for turbulent heat transfer between parallel plates ${ }^{56}$.

\subsection{Optimization of heat exchangers and heat exchanger networks}

According to the definition, the entransy dissipation-based thermal resistance of a heat exchanger is the ratio of the arithmetic temperature difference to the total heat transfer rate ${ }^{57}$, which has the following relation to the heat exchanger effectiveness.

$$
P=\frac{2}{2 R^{*}+\left(1+C^{*}\right)}
$$

where $P$ is the heat exchanger effectiveness, $R^{*}$ is the dimensionless entransy dissipation-based thermal resistance, which is the ratio of the entransy dissipation-based thermal resistance to the reciprocal of the lower heat capacity rate, and $C^{*}$ is heat capacity rate ratio. As shown in Eq. (49), the effectiveness decreases with increasing dimensionless thermal resistance. This is unlike for an entropy analysis of a cross-flow heat exchanger, where the variation of the entropy generation with the effectiveness increase is not monotonic ${ }^{57}$. That is, the entransy approach is a more effective method for optimizing cross-flow heat exchanger designs than the entropy approach.

The entransy dissipation-based thermal resistance has also been used to construct holistic constraints between the design parameters 
and system requirements for various heat transfer designs without introducing intermediate variables ${ }^{58}$. These are convenient for analyzing the heat transfer processes in complex heat transfer systems as a whole and, therefore, for optimizing the global system performance.

Fig. 10 shows a heat exchanger network with two loops connecting three heat exchangers, $\mathrm{HX}_{1}, \mathrm{HX}_{2}$ and $\mathrm{HX}_{3}$. The hot fluid flows into $\mathrm{HX}_{1}$ with inlet temperature $T_{h, i}$, heat capacity $m_{h} c_{p, h}$ and outlet temperature $T_{h, o}$. The cold fluid flows into $\mathrm{HX}_{3}$ with inlet temperature $T_{c, i}$, heat capacity $m_{c} c_{p, c}$, and outlet temperature $T_{c, o}$. The heat flow, $Q$, is transferred from the hot fluid to the cold fluid through three heat exchangers. The total heat capacity of both fluids in the internal loops is given and the optimization objective to minimize the total thermal conductance of all three heat exchangers.$$
\min \left[(k A)_{1}+(k A)_{2}+(k A)_{3}\right]
$$

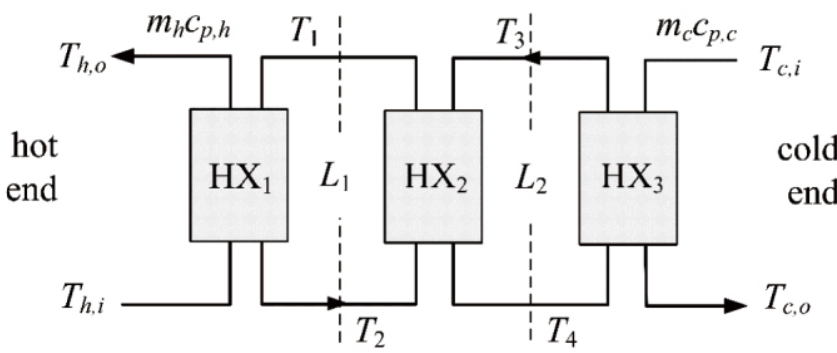

Fig.10 Multi-loop heat exchanger network ${ }^{58}$.

The conventional log-mean temperature difference method gives three heat transfer equations for the three heat exchangers and two energy conversion equations for the two internal loops for the overall constraints using the intermediate temperatures, $T_{1}, T_{2}, T_{3}$ and $T_{4}$.

$$
\begin{aligned}
& Q=(k A)_{1} \frac{\left(T_{h, o}-T_{1}\right)-\left(T_{h, i}-T_{2}\right)}{\ln \frac{T_{h o}-T_{1}}{T_{h i}-T_{2}}} \\
& Q=(k A)_{2} \frac{\left(T_{4}-T_{2}\right)-\left(T_{3}-T_{1}\right)}{\ln \frac{T_{4}-T_{2}}{T_{3}-T_{1}}} \\
& Q=(k A)_{3} \frac{\left(T_{4}-T_{c, o}\right)-\left(T_{3}-T_{c, i}\right)}{T_{4}-T_{c o}} \\
& T_{3}-T_{c i} \\
& Q=m_{1} c_{p, 1}\left(T_{2}-T_{1}\right) \\
& Q=m_{2} c_{p, 2}\left(T_{4}-T_{3}\right)
\end{aligned}
$$

In addition, the total heat capacity of the two loops fluids is

$$
m_{1} c_{p, 1}+m_{2} c_{p, 2}=g
$$

This optimization problem is a typical conditional extremum problem with the constraints in Eqs. (52) (56). A Lagrange function, $J$, is then constructed as

$$
J=\left[(k A)_{1}+(k A)_{2}+(k A)_{3}\right]+\lambda_{1}\left[Q-(k A)_{1} \frac{\left(T_{h, o}-T_{1}\right)-\left(T_{h, i}-T_{2}\right)}{\ln \frac{T_{h \rho}-T_{1}}{T_{h i}-T}}\right]
$$

$$
\begin{aligned}
& +\lambda_{2}\left[Q-(k A)_{2} \frac{\left(T_{4}-T_{2}\right)-\left(T_{3}-T_{1}\right)}{\ln \frac{T_{4}-T_{2}}{T_{3}-T_{1}}}\right]+\lambda_{3}\left[Q-(k A)_{3} \frac{\left(T_{4}-T_{c, o}\right)-\left(T_{3}-T_{c, i}\right)}{T_{4}-T_{c \rho}} \frac{T_{3}-T_{c i}}{T_{1}}\right] \\
& +\lambda_{4}\left[Q-m_{1} c_{p, 1}\left(T_{2}-T_{1}\right)\right]+\lambda_{5}\left[Q-m_{2} c_{p, 2}\left(T_{4}-T_{3}\right)\right]+\lambda_{6}\left[m_{1} c_{p, 1}+m_{2} c_{p, 2}-g\right]
\end{aligned}
$$

Making the derivative of $J$ with respect to $X_{l}\left(X_{l} \in\left\{(k A)_{m},\left(m c_{p}\right)_{i}, T_{j}\right.\right.$, $\left.\lambda_{k}\right\}$ ) equal to zero yields 9 equations. Combining these with the 6 constraints gives 15 equations to be solved to obtain the 15 unknown quantities.

The log-mean temperature difference method introduces 4 intermediate variables which increase the complexity of the system optimization analysis. The entransy-dissipation-based thermal resistance method does not need to introduce the intermediate variables which reduces the number of constraint equations. In this multi-loop heat exchanger network, the net entransy flowing into the heat exchanger network is equal to the sum of the entransy dissipation rate of each heat transfer process in the entransy balance equation which acts as the system constraint,

$$
Q\left(\frac{T_{h, i}+T_{h, o}}{2}-\frac{T_{c, i}+T_{c, o}}{2}\right)=\sum_{i=1}^{3} Q^{2} R_{i}
$$

where $R_{i}$ is the entransy-dissipation-based thermal resistance of component $i$

$$
\begin{aligned}
& R_{1}=\frac{1}{2}\left[\frac{1}{\left(m c_{p}\right)_{h}}-\frac{1}{\left(m c_{p}\right)_{1}}\right] \frac{e^{(k A)_{1}\left[\frac{1}{\left(m c_{p}\right)_{h}}-\frac{1}{\left(m c_{p}\right)_{1}}\right]}+1}{e^{(k A)_{1}\left[\frac{1}{\left(m c_{p}\right)_{h}}-\frac{1}{\left(m c_{p}\right)_{1}}\right]}-1} \\
& R_{2}=\frac{1}{2}\left[\frac{1}{\left(m c_{p}\right)_{1}}-\frac{1}{\left(m c_{p}\right)_{2}}\right] \frac{e^{(k A)_{2}\left[\frac{1}{\left(m c_{p}\right)_{1}}-\frac{1}{\left(m c_{p}\right)_{2}}\right]}+1}{e^{(k A)_{2}\left[\frac{1}{\left(m c_{p}\right)_{1}}-\frac{1}{\left(m c_{p}\right)_{2}}\right]}-1} \\
& R_{3}=\frac{1}{2}\left[\frac{1}{\left(m c_{p}\right)_{2}}-\frac{1}{\left(m c_{p}\right)_{c}}\right] \frac{e^{(k A)_{3}\left[\frac{1}{\left(m c_{p}\right)_{2}}-\frac{1}{\left(m c_{p}\right)_{c}}\right]}+1}{e^{(k A)_{3}\left[\frac{1}{\left(m c_{p}\right)_{2}}-\frac{1}{\left(m c_{p}\right)_{c}}\right]}-1}
\end{aligned}
$$

A Lagrange function, $J_{e}$, is constructed using Eqs. (59) - (61) as

$$
\begin{aligned}
J_{e} & =\left[(k A)_{1}+(k A)_{2}+(k A)_{3}\right]+\lambda_{1}\left[Q\left(\frac{T_{h, i}+T_{h, o}}{2}-\frac{T_{c, i}+T_{c, o}}{2}\right)-\sum_{i=1}^{3} Q^{2} R_{i}\right] \\
& +\lambda_{2}\left[m_{1} c_{p, 1}+m_{2} c_{p, 2}-g\right]
\end{aligned}
$$

Setting the derivative of $J_{e}$ with respect to $Y_{l}\left(Y_{l} \in\left\{(k A)_{m},\left(m c_{p}\right)_{i}\right.\right.$, $\left.\left.\lambda_{k}\right\}\right)$ equal to zero yields 5 equations. Combining these with the 2 constraints gives 7 equations that are solved for the 7 unknown quantities. Thus, the entransy method significantly reduces the number of unknown variables and constraints that simplifies the solution since the entransy dissipation based system constraint eliminates the unknown intermediate fluid temperatures and reduces 
the number of constraints for multi-component thermal systems.

\subsection{Difference between Entransy and Entropy}

Table 3 shows the differences between entransy and entropy. Entropy is a function of temperature and pressure which is a thermodynamic quantity that reflects the ability to do heat-work conversion. Entropy generation then represents the loss of ability for heat to work conversion. Entransy is a function of temperature only which is a heat transfer quantity and represents the ability of heat transfer not related to heat-work conversion. Entransy dissipation then measures the loss of ability for heat transfer.

Table 3 Comparison between entransy and entropy.

\begin{tabular}{ccc}
\hline & Entropy & Entransy \\
State quantity & $\mathrm{d} S=M C_{\mathrm{v}} \mathrm{d} T / T+R \mathrm{~d} V / V$ & $G=G(T)$ \\
& $\mathrm{d} G=M C_{\mathrm{v}} T \mathrm{~d} T$ \\
Physical meaning & Heat to work conversionability & $G=M C_{\mathrm{v}} T^{2} / 2$ (for $C_{\mathrm{v}}=$ const) \\
Process quantity & Entropy flow, $Q / T$ & Heat transfer rate ability \\
Process irreversibility & Entropy generation rate for & Entransy flow, $Q T$ \\
and optimization & thermodynamics processes & for heat transferprocesses \\
criterion & $\dot{s}_{g}=\frac{k}{T^{2}}\left(\frac{d T}{d x}\right)^{2}$ & $\dot{g}_{g}=k\left(\frac{d T}{d x}\right)^{2}$ \\
\hline
\end{tabular}

\section{Conclusions}

(1) The concept of thermomass defined by the mass-energy relation in Einstein's special relativity was used to define a phonon gas in dielectrics as a weighty, compressible fluid. Newton mechanics is then used to describe the motion of the phonon gas in a porous medium because the drift velocity of a phonon gas is normally much less than the speed of light. This means that heat has an energy-mass duality, that is, heat acts as energy during its conversion with other forms of energy but acts as a mass during its motion. In addition, unlike other forms of energy, heat is conserved during irreversible transport processes, which further demonstrates the mass nature of heat.

(2) The momentum conservation equation of a weighty phonon gas was developed as in fluid mechanics, which is the general heat conduction law degenerating into the three non-Fourier's models for various simplified conditions. The general relation between the heat flux and the temperature gradient can also be deduced from the phonon Boltzmann equation using the concept of thermomass, which is very similar to the general heat conduction law.

(3) When the heat flux in a nano-scale device is so large that the inertial force of the phonon gas cannot be neglected, Fourier's law then breaks down even for steady state conditions. In these cases, the calculated ratio of the heat flux to the temperature gradient from experimental data is the apparent thermal conductivity, which is always less than the intrinsic thermal conductivity of the material.

(4) A more general macroscopic heat conduction law for nanosystems is presented based on the phonon gas dynamics in a porous medium, where the Darcy's term represents the volume resistance and the Brinkman term represents the surface resistance respectively. As the systems get smaller, the Brinkman term becomes more important and the thermal conductivity becomes less in nanomaterials. An explicit expression is then given for the size dependent thermal conductivity of silicon nanosystems, which agrees well with experimental data for both nano-wires and films.

(5) Analogies among heat conduction, electrical conduction and fluid flow in porous medium were used to define a new quantity, the thermomass potential energy. Its simplified expression is the entransy, which is not conserved but is dissipated during the processes. The entransy dissipation is a measure of the irreversibility of heat transfer processes not related to the heat and work conversions.

(6) For heat transfer processes and simple devices, the entransy dissipation-based thermal resistance can be used as an optimization criterion to increase the energy efficiency. For complex heat transfer systems, the entransy balance equation is used as the overall system constraints to analyse or optimize the thermal performance of systems as a whole.

\section{Conflict of interest}

There are no conflicts to declare.

\section{Acknowledgment}

This work was financially supported by National Natural Science Foundation of China (No. 51356001).

\section{References}

1. C. Cattaneo, Atti. Sem. Mat. Fis. Univ. Modena., 1948, 3, 3.

2. P. Vernotte, C. R. Hebd. Seances Acad. Sci., 1958, 246, 3154-3155.

3. P. Morse, H. Feshbach, Methods of Theoretical Physics, McGrawHill, New York, 1953.

4. M. Gurtin, A. Pipkin, Arch. Ration. Mech. An., 1968, 31, 113-126.

5. B. Coleman, M. Fabrizio, D. Owen, Arch. Ration. Mech. An., 1982, 80, 135-158.

6. D. Tzou, Macro to Microscale Heat Transfer: The Lagging Behavior, Taylor \& Francis, Washington, 1997.

7. D. Joseph, L. Preziosi, Rev. Mod. Phys., 1989, 61, 41-73.

8. D. Tsai, R. MacDonald, Phys. Rev. B,1976, 14, 4714-4723.

9. D. Tzou, Annu. Rev. Heat Transfer, 1992, 4, 111-185.

10. D. Tzou, J. Heat Transfer, 1995, 117, 8-16.

11. Y. Xu, Z. Guo, Int. J. Heat Mass Transfer, 1995, 38, 2919-2922.

12. S. Brorson, J. Fujimoto, E. Ippen, Phys. Rev. Lett., 1987, 59, 1962-1968.

13. S. Lepri, R. Livi, A. Politi, Phys. Rev. Lett., 1997, 78, 1896-1899.

14. O. Narayan, S. Ramaswamy, Phys. Rev. Lett., 2002, 89, 200601.

15. S. Maruyama, Physica B, 2002, 323, 193-195.

16. R. Livi, S. Lepri, Nature, 2003, 421, 327.

17. S. Lepri, R. Livi, A. Politi, Phys. Rep., 2003, 377, 1-80.

18. A. Dhar, Adv. Phys.,2008, 57, 457-537.

19. A. Einstein, H. A. Lorentz, H. Minkowski, H. Weyl, The Principle of Relativity, Dover publications, New York, 1952.

20. Z. Guo, X. Liang, B. Cao, Proceedings of $13^{\text {th }}$ International Heat Transfer Conference, Sydney, Australia, 2006.

21. Z. Guo, B. Cao, H. Zhu, Q. Zhang, Acta. Phys.,2007, 56, 3306-3312.

22. B. Cao, Z. Guo, J. Appl. Phys., 2007, 102, 053503.

23. H. Wang, B. Cao, Z. Guo, Int. J. Heat Mass Transfer,2010, 53, 1796-1800.

24. Y. Dong, B. Cao, Z. Guo, J. Appl. Phys., 2011, 110, 063504.

25. A. Einstein, L. Infeld, The Evolution of Physics, Simon and Schuster, New York, 1961.

26. R. Feynman, R. Leighton, M. Sands, The Feynman Lecutures on Physics, Wesley, Massachusetts, 1963. 
27. H. D. Wang, Z. Y. Guo, Chin. Sci. Bull.,2010, 55(3), 1-6.

28. S. R. Groot, Thermodynamics of Irreversible Processes, NorthHolland Publishing Company, Amsterdam, 1952.

29. I. Prigogine, Introduction to Thermodynamics of Irreversible Processes. Interscience Publishers, a Division of John Wiley \& Sons, New York, 1967.

30. D. Jou, J. Casas-Vbazquez, Extended Irreversible Thermodynamics, Springer, New York, 1993.

31. D. Nield, A. Bejan, Convection in Porous Media (3rd edition), Springer, New York, 2006.

32. V. Cimmelli, A. Sellitto, D. Jou, Phys. Rev. B, 2009, 79, 014303.

33. V. Cimmelli, A. Sellitto, D. Jou, Phys. Rev. B, 2009, 81, 054301.

34. V. Cimmelli, A. Sellitto, D. Jou, Phys. Rev. B, 2010, 82, 184302.

35. Z. Banach, W. Larecki, J. Phys. A: Math. Theor, 2008, 41, 375502.

36. R. Guyer, J. Krumhans1, Phys. Rev., 1966, 148, 766-778.

37. R. Hardy, D. Albers, Phy. Rev., 1974, 10, 3546-3551.

38. W. S. Jiaung, J. R. Ho, Phys. Rev. E, 2008, 77, 066710.

39. J. Sussmann, A. Thellung, Proc. Phys. Soc., 1963, 81, 1122-1130.

40. S. Chapman, T. Cowling, Mathematical Theory of Non-Uniform Gases (3rd edition). Cambridge University Press, Cambridge, 1970.

41. M. T. Yin, L. C. Marvin, Phys. Rev. B, 1982, 26, 3259-3272.

42. G. G. Sahasrabudhe, S. D. Lambade, J. Phys. Chem. Solids,1999,
60, 773-785.

43. P. Debye, Ann. Phys., 1912, 344, 789-839.

44. Y. Dong, Dynamical Analysis of Non-Fourier Heat Conduction and Its Application in Nanosystems, Springer, 2016.

45. Y. Hua, B. Cao, Int. J. Heat Mass Transfer,2014, 78, 755-759.

46. Y. Hua, B. Cao, Int. J. Therm. Sci., 2016, 101, 126-132.

47. Y. Dong, B. Cao, Z. Guo, Physica E, 2015, 66, 1-6.

48. Y. Dong, B. Cao, Z. Guo, Physica E, 2014, 56, 256-262.

49. A. Bejan, Entropy Generation through Heat and Fluid Flow. John Wiley \& Sons, New York, 1982.

50. A. Bejan, J. Appl. Phys., 1996, 79, 1191-1218.

51. R. Shah, T. Skiepko, ASME J. Heat Transfer, 2004, 126, 994-1002.

52. Z. Guo, H. Zhu, X. Liang, Int. J. Heat Mass Transfer, 2007, 50, 2545-2556.

53. Q. Chen, X. Liang, Z. Guo, Int. J. Heat Mass Transfer, 2013, 63, 6581.

54. Q. Chen, H. Zhu, N. Pan, Z. Guo, P. Phy. Soc. A, 2011, 467, 1012-1028.

55. J. Meng, X. Liang, Z. Li, Int. J. Heat Mass Transfer, 2005, 48, 33313337.

56. Q. Chen, J. Ren, J. Meng, Int. J. Heat Mass Transfer,2007, 50, 53345339.

57. Z. Guo, X. Liu, W. Tao, R. Shah, Int. J. Heat Mass Transfer, 2010, 53, 2877-2884.

58. Y. Xu, Q. Chen, Z. Guo, Int. J. Heat Mass Transfer, 2016, 95, 109-115. 